\title{
Reduced Order Model of Single Machine Infinite Bus Power System Using Stability Equation Method and Self-adaptive Bat Algorithm
}

\author{
D. K. Sambariya*, G. Arvind
}

Department of Electrical Engineering, Rajasthan Technical University, Kota, 324010, Rajasthan, India

Copyright (C) 2016 by authors, all rights reserved. Authors agree that this article remains permanently

open access under the terms of the Creative Commons Attribution License 4.0 International License

\begin{abstract}
In this paper, a largeorder system is reduced by using the self adaptive bat algorithm (SABA) to a reduced-order approximation. The numerator coefficients of a desired reduced-order system are optimized based on integral square error minimization as an objective function pertaining to a unit-step as input. The denominator of the reduced order model is solved by stability equation method. The efficacy of the proposed method is tested with SMIB test systems to get a corresponding reduced-order system. The results are satisfactory in terms of minimum error with the proposed method as compared to Routh approximation and Stability equation based reduced models.
\end{abstract}

Keywords Model-order Reduction, Bat Algorithm, Self Adaptive Bat Algorithm (SABA), Stability Equation Method, Integral-square Error Minimization

\section{Introduction}

High-order system (HOS) is always costly and tedious. MOR is used for simplification of complicated problems in HOS. The selection of the model reduction technique is based on stability equation method as presented in [1]. The procedure of simplification is based on using mathematical approaches. Many methods to solve HOS for reduced order model (ROM) of the system are available in literature $[2,3]$. The differentiation method for model order reduction (MOR) of the systems have been proposed in $[4,5,6]$. It follows the simple steps of differentiation of the numerator and denominator polynomials but suffers with steady state error in the response of MOR as compared to original system [7, 8]. The methods in literature are not following the stability criterion except the stability equation method $[9,10]$. However, mixed method approach is prevalent to consider the stability effect in literature where in numerator and denominator are solved by using different/distinct method as in $[11,12,13]$.
Recently, Desai and Prasad have proposed the implementation of order reduction on TMS320C54X processor using genetic algorithm [14]. It also included fourth order example and reduced to 2 nd order model. Pati at el have proposed work on sub-optimal control using model order reduction [15]. Lodhwal and Jha, have proposed the performance comparison of different type of reduced order modeling methods $[16,17]$. Model order reduction using stability equation method [18] and the continued fraction method [19] have been considered for deduction of MOR systems. Sambariya and Prasad have proposed stable reduced model of a Single Machine Infinite Bus (SMIB) Power System with Power System Stabilizer, which is a seventh order system and being reduced to $2^{\text {nd }}$ order ROM [20, 21, 22, 23].

MOR is used in industrial applications. The selection of reduction technique is based on the closeness of the reduced order models response to higher order model response [24]. In literature, many authors have considered different methods to solve these problems. The Routh approximation method have been used in $[25,26]$. Alsmadi et al have presented the application of Sylvester based model order reduction for a multi-input multi-output (MIMO) power system [27]. It is based on the preservation of stability by retaining dominant poles and minimization of steady-state error. The application of particle swarm optimization (PSO) is examined in [25] by minimizing integral of square error of the response of original and reduced system. The genetic algorithm (GA) have been considered to determine the free coefficients of numerator and denominator of discrete transfer function in [28]. Soloklo have presented the application of harmony search algorithm with multi-objective function for determining the lower order model of HO systems [29]. The application of Hermite polynomials with GA is presented for ROM of large systems in [30]. The stable reduced order modeling for linear time invariant systems is presented in $[17,22]$. The application of cuckoo search is considered for deriving reduced order system of HOSs [31].

In this paper, the application of stability equation method is presented for driving reduced order model of the higher or- 
der linear time invariant systems. The statement of problem is presented in section 2 . The stability equation method is described in section 3. The self-adaptive bat algorithm (SABA) is described in section 4 . The system under consideration and the reduced order models are described in section 5. The results on subject of step input signal are discussed in this Section. Finally the paper is concluded in section 7 and followed by references.

\section{Methodology for ROM}

Consider a high order transfer function of a system represented as in Eqn. 1.

$$
G(s)=\frac{\sum_{i=0}^{n-1} b_{i} s^{i}}{\sum_{i=0}^{n} a_{i} s^{i}}
$$

where, the $G(s)$ represents a high order system with the order of $n$. The purpose of paper is to reduce the order of such high order system to $r$. The reduced order model may be represented as in Eqn. 2.

$$
R(s)=\frac{\sum_{j=0}^{r-1} d_{j} s^{j}}{\sum_{j=0}^{r} c_{j} s^{j}}
$$

where, $a_{i}, b_{i}, c_{j}$ and $d_{j}$ are the scalar constants of original high order system and the reduced order system. The objective is to find a reduced $r^{\text {th }}$ order system model $R(s)$ such that it retains the important properties of $G(s)$ for the same types of inputs.

\section{Stability equation method}

In this technique, the transfer function of reduced orders are obtained directly from the pole zero patterns of the stability equations of the original transfer function of system $[19,20]$. Thus order of the stability equations of transfer function can be reduced [31]. Assuming, a high order transfer function the system is as shown in Eqn. 3.

$$
\begin{gathered}
G(s)=\frac{b_{m} s^{m}+b_{m-1} s^{m-1}+\ldots . b_{1} s+b_{0}+b_{o}}{a_{n} s^{n}+a_{n-1} s^{n-1}+\ldots . a_{1} s+a_{0}} \\
G(s)=\frac{N(s)}{D(s)}
\end{gathered}
$$

The Eqn. 4 is the symbolic representation of Eqn. 3. The $N(s)$ and $D(s)$ are the numerator and the denominator of $G(s)$, respectively. The order of $D(s)$ is $n$ and the order of $N(s)$ is $\mathrm{m}$ such that the $n>m$.

\subsection{Separation of even,odd parts and reduction}

The numerator and the denominator of Eqn. 4 are separated in even and odd parts. The even and odd polynomials of numerator may be represented by $N_{e}(s)$ and $N_{o}(s)$, respectively if the even and odd parts are subscribed by $e$ and $o$, respectively. Similarly, the even and odd polynomials of denominator may be represented by $D_{e}(s)$ and $D_{o}(s)$, respectively. Therefore, the system in Eqn. 4 may be represented as following:

$$
\begin{gathered}
G(s)=\frac{N_{e}(s)+N_{o}(s)}{D_{e}(s)+D_{o}(s)} \\
G(s)=\frac{\sum_{i=0,2,4}^{m} b_{i} s^{i}+\sum_{i=1,3,5}^{m} b_{i} s^{i}}{\sum_{i=0,2,4}^{n} a_{i} s^{i}+\sum_{i=1,3,5}^{n} a_{i} s^{i}} \\
N_{e}(s)=\sum_{i=0,2,4}^{m} b_{i} s^{i} \\
N_{o}(s)=\sum_{i=1,3,5}^{m} b_{i} s_{i} \\
D_{e}(s)=\sum_{i=0,2,4}^{n} a_{i} s_{i} \\
D_{o}(s)=\sum_{i=1,3,5}^{n} a_{i} s_{i}
\end{gathered}
$$

The roots of $N_{e}(s)$ and $D_{e}(s)$ are called zeros $z_{i}(s)$ and that of $N_{o}(s)$ and $D_{o}(s)$ are called poles $p_{i}(s)$. In this method, a polynomial is reduced by successively discarding the less significant factors. Let us illustrate the method by reducing the denominator, the numerator is reduced similarly and the ratio of reduced numerator and denominator gives the reduced order model. The denominator is separated as following:

$$
D(s)=D_{e}(s)+D_{o}(s)
$$

where,

$$
\begin{aligned}
& D_{e}(s)=a_{0}+a_{2} s^{2}+a_{4} s^{4}+\cdots \\
& D_{o}(s)=a_{1}+a_{3} s^{3}+a_{5} s^{5}+\cdots
\end{aligned}
$$

The Eqn. 9 may be written as the following:

$$
\begin{aligned}
& D_{e}(s)=a_{0} \prod_{i=1}^{k_{1}}\left(1+\frac{s^{2}}{z_{i}^{2}}\right) \\
& D_{o}(s)=a_{1} s \prod_{i=1}^{k_{2}}\left(1+\frac{s^{2}}{p_{i}^{2}}\right)
\end{aligned}
$$

where $k_{1}$ and $k_{2}$ are integer parts of $n / 2$ and $(n-2) / 2$ respectively and $z 12<p 12<z 22<p 22 \ldots$ by discarding the factors with larger magnitude of $z_{i}$ and $p_{i}$, the reduced stability equations of desired order $r$ become

$$
\begin{aligned}
& D_{e r}(s)=a_{o} \prod_{i=1}^{r_{1}}\left(1+\frac{s^{2}}{z_{i}^{2}}\right) \\
& D_{o r}(s)=a_{1} \prod_{i=1}^{r_{2}}\left(1+\frac{s^{2}}{p_{i}^{2}}\right)
\end{aligned}
$$

where $r_{1}$ and $r_{2}$ are the integer parts of $r / 2$ and $(r-1) / 2$, respectively. The reduced denominator of the system is given as in Eqn. 12

$$
D_{r}(s)=D_{e r}(s)+D_{o r}(s)
$$

The numerator of the reduced system may be represented as in following Eqn. 13.

$$
N_{r}(s)=N_{e r}(s)+N_{o r}(s)
$$


Similarly, the complete model of the $r^{t h}$ order reduced model may be represented as in Eqn. 14.

$$
R(s)=\frac{N_{e r}(s)+N_{o r}(s)}{D_{e r}(s)+D_{o r}(s)}
$$

It may be noted that poles and zeros with smaller magnitude are more dominant than those poles or zeros with larger magnitudes. The poles or zeros with larger magnitudes are discarded in this technique. Thus the reduced order models preserve the dominant performance of the original system.

\section{On self-adaptive bat algorithms}

The bat algorithm has been introduced by Yang in 2010 [32]. It is based on the behavioral reaction of the microbats to the echolocation based orientation and prey searching. In case of the bat algorithm in [33], the main strategic parameters was the loudness and the pulse rate associated to the microbats. The pulse rate regulates an improvement of the best solution while the loudness affects the acceptance of the best solutions. These parameters were kept constant during the iterative search operation of the bat algorithm. The selection of these parameters was a tedious and time-consuming task. In the proposed self-adaptive bat algorithm (SABA), the parameters are adaptive as introduced by Fister in [34]. It serves two purposes (i) initial guess of parameters, (ii) the parameter updating, during computational phase. The self-adaptive process enables the control parameters to be changed during the iterative process to select best solutions [35].

The bat algorithm is a population-based algorithm where each microbat represents the candidate solutions. The microbats/candidate solutions may be represented as a vector, $p_{i}=\left(p_{i 1}, p_{i 2}, \cdots p_{i D}\right)^{T}$ for $i=1,2, \cdots, N_{p}$ with real valued $p_{i j}$ in the interval $p_{i j} \in\left[p_{l b}, p_{u b}\right]$. The $p_{l b}$ and $p_{u b}$ represents the lower and upper bounds of the particles, and $N_{p}$ refers to the size to the population of the candidates [36]. The process of the bat algorithm mainly consists of (i) initialization, (ii) variation operation, (iii) local search and evolution of solution, and (v) the replacement [37].

In the initialization process, two main tasks took place such as, (i) parameter initialization of the bat algorithm i.e. set value of pulse rate, loudness and frequency, etc., (ii) random generation of bat population. The best solution in initialization is determined. In variation operator, the virtual bats move according to the rate of echolocation. The current best solution is improved by the random walk in the local search $[38,39]$.

The behavior of updating is similar to the simulated annealing [40], where in the new solutions are selected with the probability of acceptance [41]. In variation operator, the virtual bats move towards the best current position according to the following Eqn. 15- Eqn. 17.

$$
\begin{gathered}
f_{i}=f_{\min }+\left(f_{\max }-f_{\min }\right) \beta \\
v_{i}^{t}=v_{i}^{t-1}+\left(v_{i}^{t-1}-p^{\prime}\right) f_{i} \\
p_{i}^{t}=p_{i}^{t-1}+p_{i}^{t}
\end{gathered}
$$

The $\beta$ represents the random number referring to the Gaussian distribution with zero mean and a standard deviation as unity. The best solution is updated as in Eqn. 18 .

$$
p^{t}=\text { best }+\varepsilon A_{i}^{t} \beta
$$

The $\epsilon$ refers to a scaling factor while the $A_{i}^{t}$ represents the loudness of the associated $i^{t h}$ microbat at instant. The local search is started with $r_{i}$ as the probability of the pulse rate. The probability of accepting the new best solutions depends on the loudness $A_{i}$. The general phenomena of operation of the bat algorithm refer to increase in pulse rate, $r_{i}$ and decrease in loudness $A_{i}$, on movement of bat nearer to the prey [42]. This behavior of microbats can be well defined in terms of following Eqn. 19 - 20.

$$
\begin{gathered}
A_{i}^{t+1}=\alpha A_{i}^{t} \\
r_{i}^{t}=r_{i}^{0}\left(1-e^{(-\gamma \varepsilon)}\right)
\end{gathered}
$$

Where, $\alpha$ and $\gamma$ are the constants. The parameter controls the rate of convergence of the bat algorithm, and $\gamma$ plays similar behavior as the cooling factor in simulated annealing algorithm $[39,43]$.

It could be easy to say that the bat algorithm in early applications as presented in $[38,40]$, was the reflection of behavior of PSO and SA algorithm. The local search behavior refers to PSO behavior, while diversity is associated to SA algorithm in bat algorithm. The exploitation (local search) is associated with the parameter, pulse rate and the exploration to loudness. It means the bat algorithm is able exploitation and exploration during the search process.

The need of self-adaptive behavior of the bat algorithm is to make it free from the initial parameter settings and updating of these parameters during the search process [34]. Therefore, the control parameters, pulse rate and loudness are set to self-adaptive to make the self-adaptive bat algorithm (SABA). It means the candidate solution represented by $p_{i}^{t}=p_{i 1}^{t}, p_{i 2}^{t}, \cdots p_{i D}^{t}$ needs to be augmented with inclusion of pulse rate $\left(r^{t}\right)$ and loudness $\left(A^{t}\right)$. The modified representation of candidate solutions may be presented as $p_{i}^{t}=\left(p_{i 1}^{t}, p_{i 2}^{t}, \cdots p_{i D}^{t} ; A^{t}, r^{t}\right)^{T}$ for $i=1,2, \cdots, N_{p}$. These control parameters are modified as by following Eqn. 21 22.

$$
\begin{gathered}
A^{t+1}=\left\{\begin{array}{l}
A_{l b}^{t}+\operatorname{rand}_{0}\left(A_{u b}^{t}-A_{l b}^{t}\right) \text { if } \text { rand }_{1} \leq \tau_{1} \\
A^{t} \quad \text { otherwise }
\end{array}\right. \\
r^{t+1}=\left\{\begin{array}{l}
r_{l b}^{t}+\operatorname{rand}_{2}\left(r_{u b}^{t}-r_{l b}^{t}\right) \text { if } \text { rand }_{3} \leq \tau_{2} \\
r^{t} \quad \text { otherwise }
\end{array}\right.
\end{gathered}
$$

The values of $A^{t}$ and $r^{t}$ are selected in the range of 0 and 1 .

\section{System and reduced order model}

\subsection{ROM using SE method}

Consider a $7^{\text {th }}$ order single-machine infinite-bus (SMIB) power system developed in [17, 21, 23] and presented in Eqn. 23

$$
G_{7}(s)=\frac{\left[\begin{array}{l}
2000 s^{6}+121700 s^{5}+1211000 s^{4} \\
+7454000 s^{3}+58270000 s^{2} \\
+31560000 s+2075000
\end{array}\right]}{\left[\begin{array}{l}
s^{7}+65.85 s^{6}+984.2 s^{5}+12130 s^{4} \\
+97300 s^{3}+429400 s^{2} \\
+2188000 s+999500
\end{array}\right]}
$$




\subsubsection{Reduction of numerator}

The $6^{\text {th }}$ order numerator of the system under consideration is shown in Eqn. 24.

$$
N_{6}(s)=\left\{\begin{array}{l}
2000 s^{6}+121700 s^{5}+1211000 s^{4} \\
+7454000 s^{3}+58270000 s^{2} \\
+31560000 s+2075000
\end{array}\right.
$$

The even and odd parts of the numerator polynomial is separated and presented in Eqn. 25.

$$
\begin{aligned}
& N_{6}(s)=\left\{\begin{array}{l}
\underbrace{2000 s^{6}+1211000 s^{4}+58270000 s^{2}+2075000}_{\text {even }} \\
+\underbrace{121700 s^{5}+7454000 s^{3}+31560000 s}_{\text {odd }}
\end{array}\right. \\
& N_{e}(s)=2000 s^{6}+1211000 s^{4}+58270000 s^{2}+2075000 \\
& N_{o}(s)=121700 s^{5}+7454000 s^{3}+31560000 s
\end{aligned}
$$

Step-1: Driving the $6^{\text {th }}$ order to $4^{\text {th }}$ order reduced order of numerator polynomial. The reduction process of even powers of $s$ polynomial in numerator is shown in Eqn. 26. The reduction of even polynomial is shown in Eqn. 26 and the odd polynomial is shown in Eqn. 27.

$$
\begin{aligned}
N_{e}(s) & =2000 s^{6}+1211000 s^{4}+58270000 s^{2}+2075000 \\
= & 1211000 s^{4}(1+\underbrace{\frac{2000}{1211000}}_{0.001651528} s^{2})+2075000(1+\underbrace{\frac{58270000}{2075000}}_{28.0819277} s^{2}) \\
= & 1211000 s^{4}+58270000 s^{2}+2075000 \\
N_{o}(s) & =121700 s^{5}+7454000 s^{3}+31560000 s \\
& =7454000 s^{3}(1+\underbrace{\frac{121700}{7454000}}_{=0.0163268} s^{2})+31560000 s \\
& =7454000 s^{3}+31560000 s
\end{aligned}
$$

The $4^{\text {th }}$ order reduced order numerator polynomial is given in Eqn. 28.

$$
N_{4}(s)=\left\{\begin{array}{l}
1211000 s^{4}+7454000 s^{3}+58270000 s^{2} \\
+31560000 s+2075000
\end{array}\right.
$$

Step-2: Reduction of numerator polynomial from $4^{\text {th }}$ order to $2^{\text {nd }}$ order polynomial.

$$
\begin{aligned}
N_{4}(s) & =\left\{\begin{array}{l}
\underbrace{1211000 s^{4}+58270000 s^{2}+2075000}_{\text {odd }} \\
+\underbrace{7454000 s^{3}+31560000 s}_{\text {even }} \\
=\left\{\begin{array}{l}
58270000 s^{2}(1+\underbrace{\frac{1211000}{58270000}}_{=0.02078256} s^{2})+2075000 \\
+31560000 s(1+\underbrace{\frac{7454000}{31560000}}_{0.23618504} s^{2}
\end{array}\right)
\end{array}\right.
\end{aligned}
$$

Step-3: Reduction of numerator polynomial from $2^{\text {nd }}$ order to $1^{\text {st }}$ order polynomial in Eqn. 30 .

$$
\begin{aligned}
& N_{2}(s)=58270000 s^{2}+31560000 s+2075000 \\
& N_{1}(s)=2075000+31560000 s
\end{aligned}
$$

\subsubsection{Reduction of denominator}

The denominator of original system presented in Eqn. 23 is shown in the following Eqn. 31.

$$
D_{7}(s)=\left\{\begin{array}{l}
s^{7}+65.85 s^{6}+984.2 s^{5}+12130 s^{4} \\
+97300 s^{3}+429400 s^{2} \\
+2188000 s+999500
\end{array}\right.
$$

The even and odd terms of the expression in above Eqn. 31 can be represented as in following Eqn. 32.

$$
\begin{aligned}
& D_{o}(s)=s^{7}+984.2 s^{5}+97300 s^{3}+2188000 s \\
& D_{e}(s)=65.85 s^{6}+12130 s^{4}+429400 s^{2}+999500
\end{aligned}
$$

Step-1: Reduction of $7^{\text {th }}$ order denominator polynomial to $5^{\text {th }}$ order as following:

$$
\begin{aligned}
D_{e}(s) & =65.85 s^{6}+12130 s^{4}+429400 s^{2}+999500 \\
& =12130 s^{4}(1+\underbrace{\frac{65.85}{12130}}_{0.0054286} s^{2})+429400 s^{2}+999500 \\
& =12130 s^{4}+429400 s^{2}+999500 \\
D_{o}(s) & =s^{7}+984.2 s^{5}+97300 s^{3}+2188000 s \\
& =984.2 s^{5}(1+\underbrace{\frac{1}{984.2}}_{0.001016} s^{2})+97300 s^{3}+2188000 s \\
& =984.2 s^{5}+97300 s^{3}+2188000 s
\end{aligned}
$$

In this way the reduced $5^{t h}$ order model of denominator polynomial can be presented as following in Eqn. 35

$$
D_{5}(s)=\left\{\begin{array}{l}
984.2 s^{5}+97300 s^{3}+2188000 s \\
+12130 s^{4}+429400 s^{2}+999500
\end{array}\right.
$$

Step-2: Reduction of $5^{\text {th }}$ order polynomial to $3^{\text {rd }}$ order polynomial.

The odd and even terms of the powers of $s$ are shown as following in Eqn. 36.

$$
\begin{aligned}
& D_{o}(s)=984.2 s^{5}+97300 s^{3}+2188000 s \\
& D_{e}(s)=12130 s^{4}+429400 s^{2}+999500
\end{aligned}
$$

The odd part is reduced by using SE method as in Eqn. 37.

$$
\begin{aligned}
D_{o}(s) & =984.2 s^{5}+97300 s^{3}+2188000 s \\
& =984.2 s^{5}+97300 s^{3}+2188000 s \\
& =97300 s^{3}(1+\underbrace{\frac{984.2}{97300}}_{0.010115} s^{2})+2188000 s \\
& =97300 s^{3}+2188000 s
\end{aligned}
$$

The even part is reduced by using SE method as in Eqn. 38.

$$
\begin{aligned}
D_{e}(s) & =12130 s^{4}+429400 s^{2}+999500 \\
& =429400 s^{2}(1+\underbrace{\frac{12130}{429400}}_{0.02824872} s^{2})+999500
\end{aligned}
$$


The combination of Eqn. 37 and Eqn. 38 gives the $3^{\text {rd }}$ order reduced denominator polynomial being presented in Eqn. 39.

$$
D_{3}(s)=97300 s^{3}+429400 s^{2}+2188000 s+999500
$$

Step-3: Reduction of $3^{\text {rd }}$ model to $2^{\text {nd }}$ order Separating the even and odd terms for application of SE method as following:

$$
\begin{aligned}
D_{3}(s) & =429400 s^{2}+999500+97300 s^{3}+2188000 s \\
& =429400 s^{2}+999500+2188000 s(1+\underbrace{\frac{97300}{2188000}}_{0.04446984} s^{2}) \\
& =429400 s^{2}+999500+2188000 s
\end{aligned}
$$

In this way the $2^{\text {nd }}$ order reduced denominator polynomial can be represented as following in Eqn. 41.

$$
D_{2}(s)=429400 s^{2}+2188000 s+999500
$$

The reduced $3^{r d}$ order polynomial of the original model using the Eqn. 30 and Eqn. 39 and dividing by 97300 is presented in following Eqn. 42.

$$
R_{3}(s)=\frac{598.8694 s^{2}+324.3576 s+21.3257}{s^{3}+4.4131 s^{2}+22.4871 s+10.2723}
$$

The reduced $2^{\text {nd }}$ order polynomial of the original model using the Eqn. 30 and Eqn. 41 and dividing by 429400 is presented in following Eqn. 43.

$$
R_{2}(s)=\frac{73.4979 s+4.8323}{s^{2}+5.0955 s+2.3277}
$$

\subsection{ROM in literature}

The reduced model of $7^{\text {th }}$ order SMIB power system developed using Routh approximation method [21] and presented in Eqn. 23 is given as following for ready reference in Eqn. 44.

$$
R_{2}(s)=\frac{81.984 s+5.390}{s^{2}+5.684 s+2.596}
$$

\subsection{ROM using SE and BA}

The application of the bat algorithm is used to determine the free elements of the numerator while keeping denominator as found by stability equation method. The problem is considered as optimization with minimization of the integral of square method and defined as in Eqn. 45. The $T_{\text {sim }}$ represents the settling time. The signal of original system and reduced system are represented by $y_{o}$ and $y_{r}$, respectively. The free elements in numerator are $N_{1}$ and $N_{2}$ and are mentioned in Fig. 1. The bounds for these parameters are selected as $0.01 \leq N_{1} \leq 100$, and $0.01 \leq N_{2} \leq 20$. The bat algorithm is forced to search optimal values of these parameters within 100 iterations. The fitness function plot during optimization in terms of value verses iterations is shown in Fig. 2. The optimal values of $N_{1}$ and $N_{2}$ are found as 72.3394 and 4.8324 , respectively. Therefore, the $2^{\text {nd }}$ order reduced model with bat algorithm and stability equation method can be represented as in Eqn. 46.

$$
\text { errorsignal }=\int_{0}^{T_{s i m}}\left|y_{o}(t)-y_{r}(t)\right|^{2} d t
$$

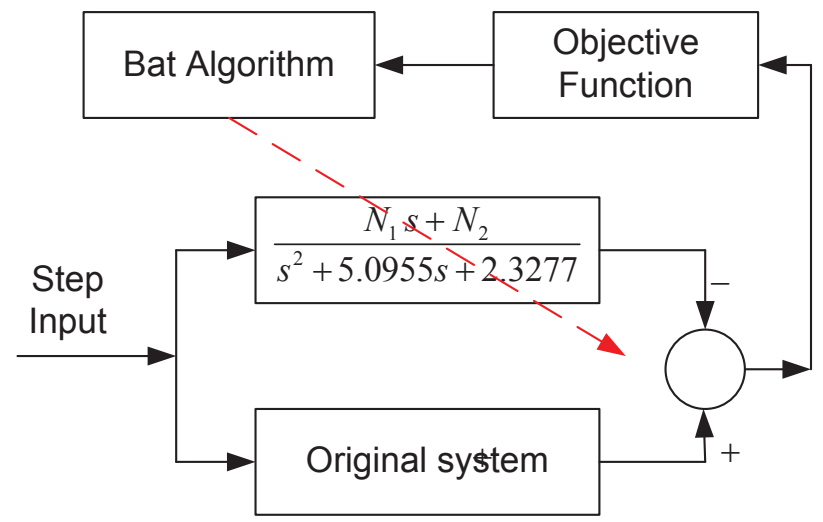

Figure 1. Scheme of determining free coefficients of numerator using bat algorithm

$$
R_{2}(s)=\frac{72.3394 s+4.8324}{s^{2}+5.0955 s+2.3277}
$$

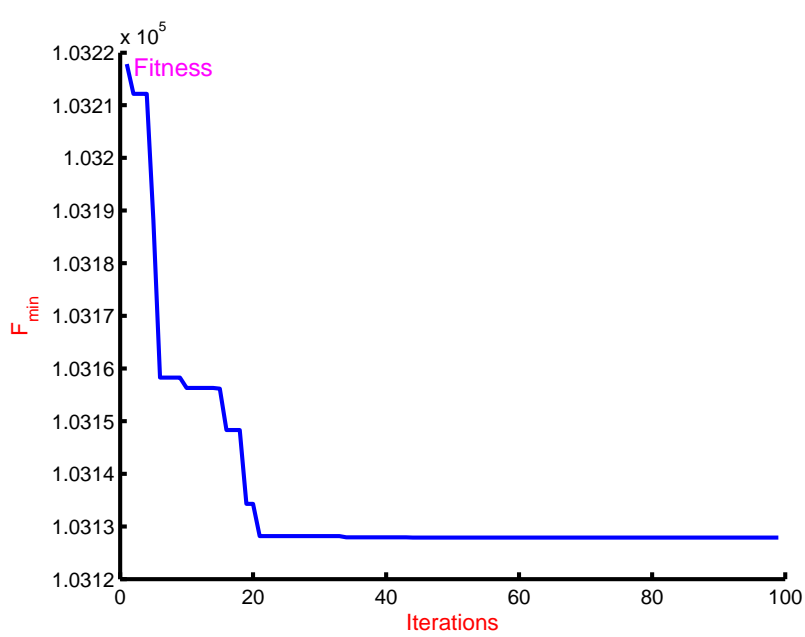

Figure 2. Plot for fitness function with iterations

\section{Results and discussion}

In this section, the study of the original system and its reduced models is being carried out. The systems are subjected to step input and the out response behaviour is analyzed using performance indices. The step response of the original system (Eqn. 23), ROM by Routh approximation (Eqn. 44 [21]), stability equation method based (Eqn. 43 [23]) and proposed stability equation method and bat algorithm is carried out. The comparative responses are shown in Fig. 3 and to make clearer, the reduced model responses are compared in Fig. 4. The responses due to reduced models are not clearly distinguishable; therefore, the performance based study of the responses are carried out. These performance indices are integral time weighted error (ITAE), integral absolute error (IAE) and integral of squared error (ISE) as defined by Eqns. 47 - 49, respectively. The detail on performance indices may be reffered in [38, 44, 45, 46, 47]. The values of these performance indices are included in Table 1. 
Table 1. Comparison of performance indices of the reduced models

\begin{tabular}{lccc}
\hline System & ITAE & IAE & ISE \\
\hline SE-BA-ROM (Proposed) & 198.61 & 109.31 & 5275.2 \\
Stability Eqn. [23] & 198.79 & 109.41 & 5275.8 \\
Routh Aprrox. [21] & 198.76 & 109.26 & 5242.9 \\
\hline
\end{tabular}

The ITAE value with proposed SE-BA-ROM is 198.61 is comparatively lower in magnitude as compared to that of with SE-MOR [23] and RA-MOR [21] as shown in Table 1. The value of IAE and ISE with proposed SE-BA-ROM are 109.31 and 5275.2, respectively and are lesser as compared to SE-MOR [23] and RA-MOR [21]. It is clear that the response of the proposed model is with minimum values of performance indices; proving its better behaviour as compared to other reduced models. However, it can be observed that the numeral values of performance indices are not having great difference. The overshoot with SE-BA-ROM is reduced as compared to SE-MOR [23] and RA-MOR [21] and is shown in Fig. 4.

$$
\begin{aligned}
I T A E & =\int_{0}^{T_{s i m}} t\left|y_{o}(t)-y_{r}(t)\right| d t \\
I A E & =\int_{0}^{T_{\text {sim }}}\left|y_{o}(t)-y_{r}(t)\right| d t \\
I S E & =\int_{0}^{T_{\text {sim }}}\left|y_{o}(t)-y_{r}(t)\right|^{2} d t
\end{aligned}
$$

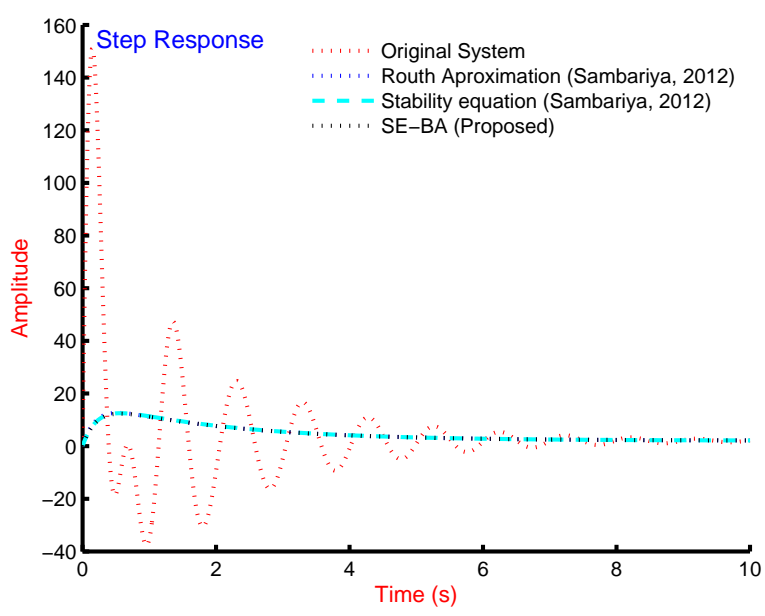

Figure 3. Step response of original system and reduced models

\section{Conclusion}

A bat algorithm based model order reduction is carried out with minimization of ISE pertaining to a unit step input. The optimization process is for bounded constraints resticted to numerator free coefficients while the denominator is solved by stability equation method. The performance of the SEBA-ROM is compared to Routh approximation method and

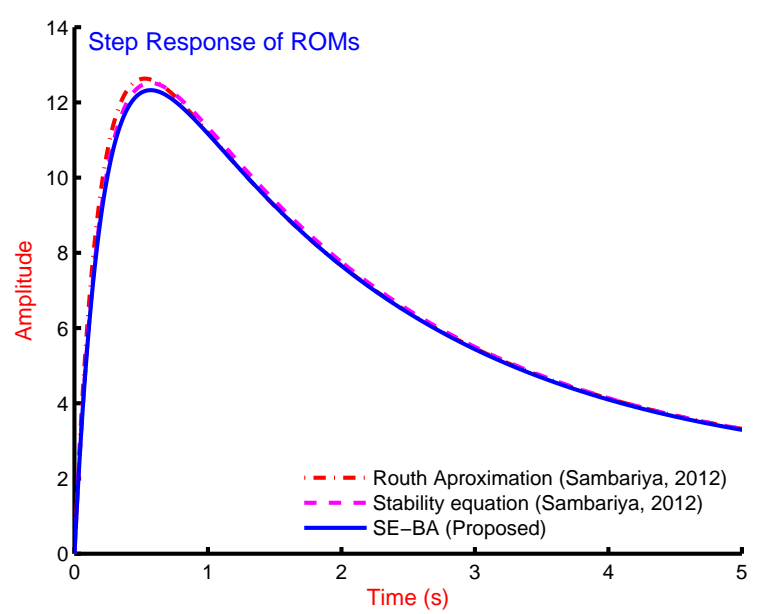

Figure 4. Step response comparison of reduced models

stability equation method in terms of performance indices. The values of ITAE, IAE and ISE reveals its better performance as compared to others in literature.

\section{REFERENCES}

[1] M. Hutton and B. Friedland, "Routh approximations for reducing order of linear, time-invariant systems," IEEE Transactions on Automatic Control, vol. 20, no. 3, pp. 329337, Jun 1975. [Online]. Available: http://dx.doi.org/10. 1109/TAC.1975.1100953

[2] R. K. Appiah, "Linear model reduction using hurwitz polynomial approximation," International Journal of Control, vol. 28, no. 3, pp. 477488, 1978. [Online]. Available: http:// dx.doi.org/10.1080/00207177808922472

[3] Y.-L. Jiang and H.-B. Chen, "Time domain model order reduction of general orthogonal polynomials for linear inputoutput systems," IEEE Transactions on Automatic Control, vol. 57, no. 2, pp. 330343, Feb 2012. [Online]. Available: http://dx.doi.org/10.1109/TAC.2011.2161839

[4] P.-O. Gutman, C.Mannerfelt, and P.Molander, "Contributions to the model reduction problem," IEEE Transactions on Automatic Control, vol. 27, no. 2, pp. 454455, Apr 1982. [Online]. Available: http://dx.doi.org/10.1109/TAC. 1982.1102930

[5] D. K. Sambariya and H. Manohar, "Preservation of stability for reduced order model of large scale systems using differentiation method," British Journal of Mathematics \& Computer Science, vol. 13, no. 6, pp. 117, 2016. [Online]. Available: http://dx.doi.org/10.9734/BJMCS/2016/23082

[6] H. Manohar and D. K. Sambariya, "Model order reduction of mimo system using differentiation method," in 10th International Conference on Intelligent Systems and Control (ISCO 2016), vol. 2, 2016, pp. 347351.

[7] D. K. Sambariya and R. Prasad, "Differentiation method based stable reduced model of single machine infinite bus system with power system stabilizer," International Journal of Applied Engineering Research, vol. 7, no. 11, pp. 2116 2120, 2012. [Online]. Available: http://gimt.edu.in/clientFiles/ FILE REPO/2012/NOV/24/1353741189722/202.pdf. 
[8] T. Lucas, "Differentiation reduction method as a multipoint pade approximant," Electronics Letters, vol. 24, no. 1, pp. 60 61, Jan 1988.

[9] T. Lucas, "A tabular approach to the stability equation method," Journal of the Franklin Institute, vol. 329, no. 1, pp. 171 180, 1992. [Online]. Available: http://www. sciencedirect.com/science/article/pii/001600329290106Q

[10] D. K. Sambariya and G. Arvind, "High order diminution of lti system using stability equation method," British Journal of Mathematics \& Computer Science, vol. 13, no. 5, pp. 115, 2016. [Online]. Available: http://dx.doi.org/10.9734/BJMCS/ $2016 / 23243$

[11] Y. Shamash, "Model reduction using the routh stability criterion and the pad approximation technique," Interna- tional Journal of Control, vol. 21, no. 3, pp. 475 484, 1975. [Online]. Available: http://dx.doi.org/10.1080/ 00207177508922004

[12] V. Krishnamurthy and V. Seshadri, "Model reduction using the routh stability criterion," Automatic Control, IEEE Transactions on, vol. 23, no. 4, pp. 729731, Aug 1978. [Online]. Available: http://dx.doi.org/10.1109/TAC. 1978.1101805

[13] A. Sikander and R. Prasad, "Linear time-invariant system reduction using a mixed methods approach," Applied Mathematical Modelling, vol. 39, no. 16, pp. 4848 4858, 2015. [Online]. Available: http://www.sciencedirect. com/science/article/pii/S0307904X15002504

[14] S. R. Desai and R. Prasad, "Implementation of order reduction on tms320c54x processor using genetic algorithm," in Emerging Research Areas and 2013 International Conference on Microelectronics, Communications and Renewable Energy (AICERA/ICMiCR), 2013, pp. 16. [Online]. Available: http:// dx.doi.org/10.1109/AICERA-ICMiCR.2013.6576027

[15] A. Pati, A. Kumar, and D. Chandra, "Suboptimal control using model order reduction," Chinese Journal of Engineering, vol. 2014, p. 5, 2014.

[16] P. Lodhwal and S. Jha, "Performance comparison of different type of reduced order modeling methods," in Third International Conference on Advanced Computing and Communication Technologies (ACCT, 13), April 2013, pp. 95100. [Online]. Available: http://dx.doi.org/10.1109/ ACCT.2013.25

[17] D. K. Sambariya and R. Prasad, "Stable reduced model of a single machine infinite bus power system," in 17th National Power Systems Conference (NPSC-2012), 2012, pp. 541548.

[18] S. R. Desai and R. Prasad, "A new approach to order reduction using stability equation and big bang big crunch optimization," Systems Science \& Control Engineering, vol. 1, no. 1, pp. 2027, 2013. [Online]. Available: http://dx.doi.org/ $10.1080 / 21642583.2013 .804463$

[19] T. C. Chen, C. Y. Chang, and K. W. Han, "Model reduction using the stability-equation method and the continued-fraction method," International Journal of Control, vol. 32, no. 1, pp. 8194, 1980. [Online]. Available: http://dx.doi.org/10.1080/ 00207178008922845

[20] D. K. Sambariya and R. Prasad, "Stable reduced model of a single machine infinite bus power system with power system stabilizer," in International Conference on Advances in Technology and Engineering (ICATE13), Jan 2013, pp. 110. [Online]. Available: http://dx.doi.org/10.1109/ICAdTE. 2013.6524762
[21] D. K. Sambariya and R. Prasad, "Routh approximation based stable reduced model of single machine infinite bus system with power system stabilizer," in DRDO-CSIR Sponsered: IX Control Instrumentation System Conference (CISCON 2012), 2012, pp. 8593.

[22] D. K. Sambariya and R. Prasad, "Routh stability array method based reduced model of single machine infinite bus with power system stabilizer," in International Conference on Emerging Trends in Electrical, Communication and Information Tech- nologies (ICECIT-2012), 2012, pp. 2734.

[23] D. K. Sambariya and R. Prasad, "Stability equation method based stable reduced model of single machine infinite bus system with power system stabilizer," International Journal of Electronic and Electrical Engineering, vol. 5, no. 2, pp. 101 106,2012

[24] J. Bansal, H. Sharma, and K. Arya, "Model order reduction of single input single output systems using artificial bee colony optimization algorithm," in Nature Inspired Cooperative Strategies for Optimization (NICSO 2011), ser. Studies in Computational Intelligence, D. Pelta, N. Krasnogor, D. Dumitrescu, C. Chira, and R. Lung, Eds. Springer Berlin Heidelberg, 2011, vol. 387, pp. 85100. [Online]. Available: http://dx.doi.org/10.1007/978-3-642-24094-2_6

[25] A. Sikander and R. Prasad, "Soft computing approach for model order reduction of linear time invariant systems," Circuits, Systems, and Signal Processing, vol. 34, no. 11, pp. 34713487, 2015. [Online]. Available: http://dx.doi.org/10. 1007/s00034-015-0018-4

[26] D. K. Sambariya and H. Manohar, "Model order reduction by differentiation equation method using with routh array method," in 10th International Conference on Intelligent Systems and Control (ISCO 2016), vol. 2, 2016, pp. 341346.

[27] O. M. K. Alsmadi, S. S. Saraireh, Z. S. Abo-Hammour, and A. H. Al-Marzouq, "Substructure preservation sylvesterbased model order reduction with application to power systems," Electric Power Components and Systems, vol. 42, no. 9, pp. 914926, 2014. [Online]. Available: http://dx.doi.org/10. $1080 / 15325008.2014 .903543$

[28] O. M. K. Alsmadi and Z. S. Abo-Hammour, "A robust computational technique for model order reduction of two-timescale discrete systems via genetic algorithms," Computational Intelligence and Neuroscience, vol. 2015, p. 9, 2015. [Online]. Available: http://dx.doi.org/10.1155/2015/615079

[29] H. N. Soloklo and M. M. Farsangi, "Multiobjective weighted sum approach model reduction by routh-pade approximation using harmony search," Turk J Elec Eng \& Comp Sci, vol. 21, no. 0, pp. 2283 2293, 2013. [Online]. Available: http//dx. doi.org/10.3906/elk-1112-31

[30] H. N. Soloklo, O. Nali, and M. M. Farsangi, "Model reduction by hermite polynomials and genetic algorithm," Journal of mathematics and computer science, vol. 9, no. 0, pp. 188 202, 2014.

[31] A. Sikander and R. Prasad, "A novel order reduction method using cuckoo search algorithm," IETE Journal of Research, vol. 61 , no. 2 , pp. 8390 , 2015. [Online]. Available: http://dx. doi.org/10.1080/03772063.2015.1009396

[32] X.-S. Yang, "A new metaheuristic bat-inspired algorithm," in Nature Inspired Cooperative Strategies for Optimization 
(NICSO 2010), ser. Studies in Computational Intelligence, J. Gonzlez, D. Pelta, C. Cruz, G. Terrazas, and N. Krasnogor, Eds. Springer Berlin Heidelberg, 2010, vol. 284, pp. 6574. [Online]. Available: http://dx.doi.org/10.1007/ 978-3642-12538-6 6

[33] D. K. Sambariya and H. Manohar, "Model order reduction by integral squared error minimization using bat algorithm," in IEEE Proceedings of 2015 RAECS UIET Panjab University Chandigarh 21 22nd December 2015, pp. 17.

[34] I. Fister, Y. Xin-She, S. Fong, and Z. Yan, "Bat algorithm: Recent advances," in IEEE 15th International Symposium on Computational Intelligence and Informatics (CINTI-2014), 2014, pp. 163167. [Online]. Available: http://dx.doi.org/10. 1109/cinti.2014.7028669

[35] I. Fister, S. Fong, and J. Brest, "A novel hybrid self-adaptive bat algorithm," The Scientific World Journal, vol. 2014, p. 12, 2014. [Online]. Available: http://dx.doi.org/10.1155/2014/ 709738

[36] D. K. Sambariya and R. Prasad, ”Application of bat algorithm to optimize scaling factors of fuzzy logic-based power system stabilizer for multimachine power system," Interna- tional Journal of Nonlinear Sciences and Numerical Simula- tion, vol. 17, no. 1, pp. 4153, 2016.

[37] C. Abdelghani, C. Lakhdar, A. Salem, B. M. Djameleddine, and M. Lakhdar, "Robust design of fractional order pid sliding mode based power system stabilizer in a power system via a new metaheuristic bat algorithm," in International Workshop on Recent Advances in Sliding Modes (RASM), 2015, pp. 15. [Online]. Available: http://dx.doi.org/10.1109/ rasm.2015.7154651

[38] D. K. Sambariya and R. Prasad, "Robust tuning of power system stabilizer for small signal stability enhancement using metaheuristic bat algorithm," International Journal of Electrical Power \& Energy Systems, vol. 61, no. 0, pp. 229 238, 2014. [Online]. Available: http://www.sciencedirect. com/science/article/pii/S0142061514001616

[39] S. Ylmaz and E. U. Kksille, "A new modification approach on bat algorithm for solving optimization problems," Applied Soft Computing, vol. 28, pp. 259275, 2015. [Online]. Available: http://dx.doi.org/10.1016/j.asoc.2014.11.029
[40] S. Kirkpatrick, C. Gelatt, and M. P. Vecchi, "Optimization by simulated annealing," Science, vol. 220, no. 4598, pp. 671 680, 1983.

[41] A. H. Gandomi and X.-S. Yang, "Chaotic bat algorithm," Journal of Computational Science, vol. 5, no. 2, pp. 224232, 2014. [Online]. Available: http://dx.doi.org/10.1016/j.jocs. 2013.10.002

[42] X.-S. Yang, "Bat algorithm for multi-objective optimisation," International Journal of Bio-Inspired Computation, vol. 3, no. 5, pp. 267274, 2011. [Online]. Available: http://dx.doi. org/10.1504/ijbic.2011.042259

[43] X.-B. Meng, X. Z. Gao, Y. Liu, and H. Zhang, ”A novel bat algorithm with habitat selection and doppler effect in echoes for optimization," Expert Systems with Applications, vol. 42, no. 1718 , pp. 63506364,2015 . [Online]. Available: http:// dx.doi.org/10.1016/j.eswa.2015.04.026

[44] D. K. Sambariya and R. Prasad, "Optimal tuning of fuzzy logic power system stabilizer using harmony search algorithm," International Journal of Fuzzy Systems, vol. 17, no. 3, pp. 457470, 2015. [Online]. Available: http://dx.doi. org/10.1007/s40815-015-0041-4

[45] D. K. Sambariya, "Power system stabilizer design using compressed rule base of fuzzy logic controller," Journal of Electrical and Electronic Engineering, vol. 3, no. 3, pp. 5264, 2015. [Online]. Available: http://dx.doi.org/10.11648/ j.jeee. 20150303.16

[46] D. K. Sambariya and R. Prasad, "Design of robust PID power system stabilizer for multimachine power system using HS algorithm," American Journal of Electrical and Electronic Engineering, vol. 3, no. 3, pp. 7582, 2015. [Online]. Available: http://dx.doi.org/10.12691/ajeee-3-3-3

[47] D. K. Sambariya and R. Prasad, "Evaluation of interval type-2 fuzzy membership function \& robust design of power system stabilizer for smib power system," Sylwan Journal, vol. 158, no. 5, pp. 289307, 2014. [Online]. Available: http://sylwan. ibles.org 